\title{
Sharing Situational Awareness of the 2014-2015 Highly-Pathogenic Avian Influenza Outbreak Across Government
}

\author{
Yandace K. Brown* and Teresa Quitugua
}

National Biosurelliance Integration Center, Department of Homeland Security, Washington DC, DC, USA

\section{Objective}

The National Biosurveillance Integration Center (NBIC) coordinated information sharing with the U.S. Department of Agriculture (USDA/APHIS) and the Department of Interior (DOI/ $\mathrm{NWHC}$ ) to integrate information and provide shared situational awareness of the 2014-2015 Highly Pathogenic Avian Influenza (HPAI) outbreak in the U.S. across all levels of government.

\section{Introduction}

NBIC integrates, analyzes, and shares national biosurveillance information provided from capabilities distributed across public and private sectors. The integration of information enables early warning and shared situational awareness of nationally significant biological events to inform critical decisions directing response and recovery efforts.

The 2014-2015 HPAI H5 outbreak in the U.S. was the largest HPAI outbreak in the country's history and resulted in the culling of millions of domestic birds and significant economic losses through loss wages, direct production losses, cost of recovery, consumer price increases, and trade restrictions.

NBIC worked closely with liaisons from USDA/APHIS and DOI/ NWHC over the course of the outbreak to integrate information from both agencies and open source reporting into reports and data sets providing early and sustained shared situational awareness to over 1400 federal, state, and local authorities.

\section{Methods}

NBIC collects data from numerous sources by automated and manual methods. Most information gathered is unstructured data, such as media reports and is collected, analyzed, and visualized using government funded and publically available information technology tools. Whenever possible, open source reports are verified, validated, and contextualized through consultation of reports from interagency partners, communication with interagency liaisons and other subject matter experts, or through formal interagency Requests For Information. Results of integrated, collaborative analyses of the aggregated data are communicated in reports disseminated to all levels of government through secure automated feeds to government systems, posts on government information sharing portals, or direct email.

\section{Results}

In mid-December 2014, DOI/NWHC reported the first confirmation of HPAI H5 in a captive gyrfalcon in Whatcom County, Washington at a site near an area of British Columbia, Canada where an outbreak of HPAI H5N2 occurred earlier that month. NBIC began reporting on the outbreak through the National Biosurveillance Integration System (NBIS) Monitoring List, a daily summary of high-priority events distributed to federal, state, and local partners. The next day, NBIC issued its first Biosurveillance Event Report, a more extensive report that includes assessment of potential impact and comprehensive background information.
Over the course of the outbreak, NBIC analysts communicated closely with DOI/NWHC and USDA/APHIS liaisons and continuously monitored open-source media; including state and federal government, industry, and academic reports, and updates to provide timely and accurate information to a broad range of stakeholders. NBIC integrated data made available by individual federal authorities into a single map that was updated regularly to reflect the most current information available on the locations of HPAI detections in wild, backyard, captive, and commercial birds. NBIC provided structured datasets to the Department of Health and Human Services Office of the Assistant Secretary for Preparedness and Response, which were used to generate maps for sharing within the GeoHealth platform. NBIC also worked with the Federal Emergency Management Administration to communicate situational updates to regional planning and response personnel.

By June 2015, HPAI H5 was identified in wild, captive, backyard, or commercial birds in 21 U.S. states. Detections in backyard and commercial poultry were confirmed in 15 U.S. states with over 48 million domestic birds affected.

\section{Conclusions}

NBIC's ongoing efforts to cultivate and maintain interagency relationships across the federal government to enhance biosurveillance capabilities facilitated situational awareness. NBIC provided integrated maps not available elsewhere and coordinated timely reporting shortly after initial notification by the responsible authorities. NBIC continues to coordinate with DOI/NWHC and USDA/APHIS through agency liaisons and provide situational awareness to its government partners and department leadership.

\section{Keywords}

HPAI; outbreak; situational awareness; government

\section{*Yandace K. Brown}

E-mail: Yandace.Brown@hq.dhs.gov 